Lucija Piß̌t - Čok

Ljubljana

\title{
CONTRIBUTO ALLA DIDATTICA DELLA LINGUA ITALIANA COME LINGUA SECONDA *
}

La tesi postlaurea "Contributo alla didattica della lingua italiana come lingua seconda" è frutto di esperienze raccolte dall'autrice nell'insegnamento della lingua italiana come $\mathrm{L}_{2}$, nonché sintesi riflessiva del lavoro empirico che l'educazione al bilinguismo le ispirava. L'insegnamento della lingua italiana nell'ambiente bilingue e biculturale dell'Istria deve differire dall'insegnamento dell'italiano come lingua straniera. Esso si pone finalità immediate (L'italiano è lingua veicolare) e finalità a lungo termine (L'italiano è una delle due lingue- culture del bilingue). Esso ha obiettivi cognitivi (L'italiano è scienza), sociali e etnolinguistici (L'educazione bilingue è fattore di transculturismo). L'autrice in prima persona ha avuto modo e opportunità di avvedersene, sia come collaboratrice nella stesura dei programmi per la lingua seconda, che come autrice di libri di testo per l'insegnamento di questa lingua, e relatrice in diversi tipi di aggiornamento professionale per insegnanti di lingua italiana elaborando, sperimentando e verificando le specificità dell'insegnamento della lingua italiana come $\mathrm{L}_{2}$. Il suo contributo alla teoria dell'educazione bilingue e le proposte per rendere l'insegnamento della lingua italiana come $\mathrm{L}_{2}$ efficace e valido, sono il nucleo della tesi. Esso si riflette a livello prescolare (Dal gioco alla parola), a livello di scuola elementare (Uso e consapevolezza linguistici) e a livello di scuola media superiore (Lingua - strumento di interazioni linguistiche e sociali nell'ambiente bilingue).

La parte conclusiva della tesi presenta la visione dell'educazione al bilinguismo dai primi passi alla creazione di strutture pedagogiche e sociali per una maggiore operatività e incisività dell'educazione bilingue stessa, propone ambiti di ricerca e consiglia sperimentazioni didattiche. Alla tesi, che nell'originale sloveno conta 137 cartelle dattiloscritte, vengono allegate 18 tavole di strumenti di ricerca e di elaborazione di dati.

In questa breve presentazione dell'opera vengono trattate le specificità socio- $\mathrm{e}$ psicolinguistiche dell'educazione al bilinguismo sloveno - italiano nell'Istria nonché l'applicazione dello specifico nella prassi didattica.

* La tesi postlaura è stata presentata e diffesa dall' autrice all' Università di Ljubljana, Facoltà di Filosofia in settembre 1988. La valutazione della tesi è stata effetuata da mentori: professore ordinario dr. Barica Marentic - Požarnik e professore ordinario dr. Mitja Skubic nonché dal membro della commissione professore ordinario dr. France Strmenik. Il lavoro è il risultato di studi interdisciplinari (lingua italiana-pedagogia ). 


\section{Educazione al bilinguismo e glottodidattica}

Le novità che hanno travolto la linguistica, il coinvolgimento della psicologia nello studio del linguaggio, l'applicazione di concetti sociologici nell'evento linguistico, la pedagogia moderna hanno ridimensionato l'importanza che la teoria scientifica può avere nella prassi dell'insegnamento linguistico. Concetti teorici e ricerca empirica consolidano la posizione della glottodidattica come scienza.

La didattica della lingua seconda ancora più della didattica della lingua straniera, si pone due massime di fondo:

1. l'acquisizione della seconda lingua e di tutte le lingue seguenti si basa sullo sviluppo della lingua materna, la complementa e l' approfondisce. Essa presenta dunque un lato specifico dello sviluppo linguistico globale dell'individuo;

2. l'atto linguistico è un atto sociale. La lingua come strumento di interazione sociale stabilisce rapporti e relazioni comunicativi e sociali.

La lingua è materia conoscitiva e strumento cognitivo. Acquisire una seconda lingua significa interiorizzare la competenza linguistica e sviluppare la competenza comunicativa di una seconda lingua. Allo stesso tempo vengono ulteriormente approfondite e sviluppate le competenze (linguistica e comunicativa) globali dell'individuo. Conoscere una seconda lingua significa sentire una seconda cultura, accettarla e condividerla, farne bagaglio personale. La glottodidattica è, per le finalità a cui tende e per la materia che elabora, scienza applicata interdisciplinare.

Concetti base della glottodidattica moderna,rielaborati da Robert Lado (1964) hanno subito ulteriori revisioni da parte di teorici dell'insegnamento della seconda lingua (D. Girard, 1977, L. A. Jakobovits, 1970, B. MCLaughin 1978, D. Wilkins 1973, S. D. Krashen 1981, I. A. Fishman 1978. G. Francescato 1973 e 1981, R. Titone 1971 e 1986...)

Il processo d'apprendimento della seconda lingua per W. F. Mackay, 1982 differisce da quello della lingua straniera per quattro aspetti fondamentali:

\section{Apprendimento funzionale della lingua seconda}

Il significato linguistico, il senso delle strutture che lo simbolizzano, sono per il bilingue il prodotto della sua esperienza, ed hanno non solo un significato verbale,ma anche sociale, culturale, affettivo. Ogni significato è il risultato di una grande varietà di situazioni comunicative in cui esso si concretizza.

Malgrado gli sforzi fatti dagli insegnanti per stimolare situazioni reali tramite un approccio comunicativo e con metodi funzionali, la situazione scolastica in cui l'alunno apprende la lingua straniera resta artificiale. 


\section{Acculturazione concettuale}

Il bilingue utilizza la seconda lingua per rifare l'immagine del suo universo. Mentre si integra nell'ambiente bilingue è portato a rifare le categorie che gli hanno consentito, nella sua lingua, di strutturare il suo universo concettuale.

L'alunno che inizia lo studio della lingua straniera ha già acquisito il sistema concettuale. Quando si esprime nella lingua straniera nella sua mente domina la disponibilità dei primi concetti elaborati.

\section{Comportamento globale} globale.

Il bilingue apprende la seconda lingua come componente di un comportamento

All'alunno che studia la lingua straniera si promette a titolo di ricompensa, che in un futuro lontano potrà utilizzare la lingua che studia come componente del suo comportamento sociale, culturale e linguistico.

\section{Inserimento sociale}

Il bilingue si inserisce gradatamente nell'altra comunità linguistica. Essendo ricompensato per i suoi sforzi esso irrobustisce il suo apprendimento linguistico. Piu numerosi sono gli stimoli a usare le due lingue, migliore è il processo d'acquisizione, più si concretizza il consolidamento sociale e psicologico che ne deriva.

Nell'apprendimento della lingua straniera l'insegnante crea situazioni artificiali con cui avvicinare agli alunni la comunità che la lingua rappresenta. Però nulla, se non un inserimento fisico, potrà far sentire agli alunni di essere parte integrante della comunità in questione.

Gli obiettivi a cui l'apprendimento bilingue mira sono l'acquisizione di due lingue e l'interiorizzazione di due culture. Il processo tramite il quale il singolo vuole raggiungerle è globale, formativo. Gli stimoli provenienti dall'ambiente bilingue possono o non possono convalidare l'esperienza linguistica individuale. I contenuti comunicativi si offrono al bilingue nell'ambiente circostante e dalla sua attività linguistica nascono o si creano i suoi bisogni comunicativi.

L'apprendimento scolastico deve riflettere la realtà culturale, sociale e linguistica dell'ambiente bilingue e ci si deve ritrovare. L'approccio didattico dell'educazione al bilinguismo è dunque molto diverso da quello della lingua straniera. Metodologie operative e dinamiche, attività extrascolastiche, contenuti interdisciplinari ne sono la base.

L'insegnante della lingua seconda è un operatore culturale e sociale che si fa promotore del transculturalismo e del bilinguismo. La sua preparazione professionale va completata dalla preparazione socio-e psicolinguistica, nonché da quella etnolinguistica. 
L'acquisizione del bilinguismo è un processo cognitivo e socializzante. Apprendendo a parlare interiorizziamo i valori e le esperienze conoscitivi che l'ambiente circostante ci offre, esplorando cio che ci circonda arriviamo ad esperienze proprie. Integrando $\mathrm{i}$ due processi euristici formiamo reti conoscitive che permettono l'astrazione e la concettualizzazione del messaggio linguistico. Il coinvolgimento attivo del singolo nel processo comunicativo sblocca il confronto dell'esperienza sociale collettiva con l'esperienza sociale e cognitiva individuale. Il linguaggio funge da canale e da codice.

Lo sviluppo del linguaggio individuale si presenta come acquisizione progressiva di singoli sottosistemi linguistici (interlingua). Questi sistemi sono unici e perfetti riguardo allo stato provvisorio dello sviluppo globale del singolo. Mentre esso progredisce tramite processi evolutivi, i sistemi cambiano e si arricchiscono diventando sempre più complessi. Presentano una sintesi elaborata del codice linguistico sociale e del codice linguistico personale.

Il modello linguistico sociale è strutturato di varianti e combinazioni. Esso si presenta come norma (lingua) e come realizzazione (varietà linguistiche) di uno o di piu codici linguistici. L'acquisizione plurilingue è resa possibile in alcune condizioni ambientali, che promuovono e stimolano fattori di sviluppo individuale specifico (psicolinguistico, cognitivo e sociale).

Il bambino che cresce nell'ambiente bilingue può assimilare i due codici linguistici simultaneamente o successivamente. Il rapporto tra i due codici è determinato da fattori culturali, sociali ed economici a cui l'ambiente familiare espone il bambino facendosi cosí organo di trasmissione del mondo esteriore.

Il significato del segno linguistico concepito dal bambino bilingue è neutrale. Il valore specifico gli viene trasmesso con l'esperienza altrui che si fa sua e reale nel momento della verifica. Se l'ambiente bilingue stimola all'uso delle due lingue che il bambino apprende, i suoi bisogni linguistici, la sua intenzionalità comunicativa, l'attesa realizzazione non differiscono. Essi sono indipendenti dalla lingua che il bambino sceglie per effettuare l'atto comunicativo. La posizione della sua lingua materna (prima lingua) in confronto con la lingua seconda si stabilirà conformemente alla qualità dei rapporti linguistici, al valore degli effetti comunicativi, alla complessità dei messaggi linguistici che usando le due lingue potrà realizzare.

Le tipologie del bilinguismo, fondate su criteri di valutazione della competenza bilingue in atto, cercano di definire l'allontanamento del singolo dal monolinguismo e l'avvicinamento dello stesso all'equilinguismo. Il livello della competenza bilingue si stabilisce nel campo semantico oppure in tutti i campi della competenza linguisticocomunicativa. Lo studio empirico del bilinguismo verifica l'equilibrio bilingue a diversi livelli e di diversa qualità.

L'equilibrio bilingue si può dimostrare tramite la competenza orale e quella scritta, nel campo morfosintattico e semantico ed anche in quello pragmatico, a livello di 
norma o di uso linguistico. Conservare la competenza bilingue è faticoso, richiede sforzo cognitivo e volontà, coscienza individuale e stimoli esterni. Conformemente a fattori individuali e sociali essa oscilla nella sua solidità dall'una all'altra lingua.

\section{Fattori psicolinguistici dell'acquisizione bilingue}

Le analisi dell'acquisizione bilingue spontanea hanno confermato che i processi dell'acquisizione $\mathrm{L}_{1} / \mathrm{L}_{2}$ sono analoghi e complementari. Le forme e le relazioni strutturali convergenti si acquisiscono una sola volta in una delle due lingue. Le nozioni linguistiche interiorizzate prima fungono da filtro al momento dell'acqusizione successiva. Con l'aiuto del transfer cognitivo vengono così automaticamente elaborate strutture e funzioni linguistiche convergenti nelle due lingue (teoria dell'interdipendenza linguistica, J. Cummins 1984).

L'apprendimento di una nuova lingua presenta dunque tante nozioni da interiorizzare quante sono le strutture/funzioni convergenti non acquisite in nessuna delle lingue che già conosciamo e le strutture/funzioni divergenti o specifiche che la nuova lingua possiede.

La prassi scolastica ha fatto buon uso di questa costatazione.

All'apprendimento delle lingue classiche, per esempio, si attribuiva un valore formativo dando così poca importanza al valore funzionale di una lingua. Il transfer funzionale stimola infatti l'apprendimento linguistico e lo convalida.

Generare lingua è un processo complesso. Tra l'altro esso richiede dal parlante la volontà di subire la fatica che esso cômporta. Meccanismi di difesa alleviano questa fatica. Si verifica così nell'atto di generar lingua la tendenza alla generalizzazione di regole e la formazione di analogie. L'interferenza provoca false analogie. Non tutte però sono effetto dell'interferenza. Alcune tra di esse si possono verificare in conseguenza della rigidità che il sistema metalinguistico già acquisito presenta e per la perseveranza che l'interlingua conserva. Del resto l'interferenza mitiga pure lo sforzo che nel parlante bilingue comporta lo scambio di codice.

Essa è forte e frequente, la più frequente a livello lessicale, un po' meno frequente a livello fonologico, ancora meno attiva a livello morfosintattico. Questi fenomeni psicolinguistici (la generalizzazione, l'interferenza...) permettono al parlante bilingue una migliore e più libera osservazione della lingua come sistema simbolico di regole.

Lo sforzo provocato dallo scambio di codice diventa un'abitudine, dunque un modo di reagire per comunicare. Il bambino che vive in un ambiente bilingue può effettuare il cambiamento di codice coscientemente, per volontà propria già all'età di 3 o 4 anni. Sceglie il codice per il contenuto del messaggio conformemente alla funzione e al contesto linguistici. Motivato dall'intento di voler realizzare il messaggio linguistico cambia di codice automaticamente. La traduzione fedele da una all'altra lingua invece è un'operazione linguistica e cognitiva troppo complessa, che il bambino in tenera età non è capace di effettuare. 
I vantaggi dell'acquisizione bilingue precoce e simultanea si manifestano nel modo in cui il parlante bilingue concepisce le funzioni linguistiche, interiorizza le cognizioni e diminuisce lo sforzo dello scambio di codice linguistico. Secondo la teoria dell'interdipendenza linguistica la $L_{1}$ funge da base per l'osservazione e l'acquisizione della $\mathrm{L}_{2}$.

L'educazione bilingue successiva, per risultare efficace dovrebbe aver inizio tra $i$ 7 e 12 anni. Dall'età di dodici anni in poi alcune capacità psicofisiche che favoriscono l'acquisizione bilingue perdono d'intensità. L'acquisizione della seconda lingua sia simultanea che successiva necessita di funzionalità dell'uso linguistico e di stimoli motivanti che l'ambiente bilingue offre.

\section{Fattori sociolinguistici dell'acquisizione bilingue}

La lingua è uno strumento che permette al singolo parlante di interiorizzare norme, valori, concetti sul mondo esteriore sia fisico che simbolico. Le motivazioni e gli argomenti trasmessi da altri, la verifica degli stessi nel dinamismo dei rapporti interpersonali facilitano la socializzazione del singolo. Rivivere l'esperienza altrui consolida in lui le conoscenze.

Nella famiglia bilingue il bambino simbolizza il mondo esteriore tramite l'acquisizione e l'uso delle due lingue. Nel processo cognitivo passa dall'aspetto concreto all'aspetto astratto delle cose; con l'ausilio di più significanti per un significato la sua esperienza linguistica si consolida diventando elaborata e globale. I fattori familiari e ambientali agiscono sullo sviluppo sociale del bambino determinando la relazione tra le due lingue. Tramite le due lirrgue conosce il mondo che lo circonda. Esso gli si presenta come un'unica realtà quando le funzioni conoscitive e sociali dei due codici si equivalgono. Il mondo esteriore si dimostra diviso in due realtà distinte quando i due codici si escludono o si differenziano nelle loro funzioni. L'atto linguistico per se stesso indipendentemente dal codice usato scaturisce da bisogni linguistici identici, mira alla realizzazione dello stesso scopo linguistico e si promette gli stessi effetti. La reazione, linguistica o extralinguistica qualunque essa sia, proveniente dal contesto sociale, stabilirà la qualità del contatto comunicativo e sociale che il parlante sta per instaurare.

Nell'ambiente plurilingue elementi di culture compresenti si scambiano, si mescolano e si completano. Nel processo di acculturazione e di socializzazione del singolo essi possono arricchire e approfondire oppure discriminare e offendere la sua identità personale. Il contenuto degli afflussi culturali che passano dall'ambiente circostante al singolo è determinato dalla complessità di rapporti nazionali, sociali e culturali che la società, in cui egli vive, istituisce. Processi socializzanti continui, armonici e completivi assicurano al singolo e alla società uno sviluppo normale, ricco e continuo. Tali processi permettono l'esistenza e garantiscono la prosperità del bilinguismo e del transculturalismo.

Processi socializzanti controversi, discontinui e disarmonici creano un divario tra l'identità culturale del singolo e l'identità culturale del gruppo sociale. Ne derivano la 
stratificazione sociale, i processi discriminativi e assimilativi. Nel secondo dopoguerra la discontinuità della socializzazione della lingua/cultura italiana nel territorio istroquarnerino ha provocato effetti negativi sull'identità culturale del singolo di nazionalità italiana. Il calo della popolazione italiana, causata dal grande esodo, gli afflussi di popolazione alloctona, i matrimoni misti, l'isolamento, provocato da regolamenti governativi parziali o irrealizzati, hanno spinto e spingono il gruppo nazionale italiano nella disuguaglianza nazionale e culturale. Il bilinguismo è una condizione d'obbligo per il gruppo nazionale italiano minoritario e una libera scelta per la popolazione maggioritaria. Esso è sempre di meno un valore comune e cessa di essere un'opportunità qualitativa per tutti. La società mistilingue e pluriculturale diventa così sempre più nazionalmente stratificata, la sua fisionomia culturale perde d'originalità e il suo valore autoctono si deforma.

\section{Bilinguismo istituzionalizzato nel territorio mistilingue dell'Istria slovena}

La volontà politica della Slovenia di conservare o tutelare il bilinguismo nel territorio mistilingue dell'Istria non basta. Il bilinguismo individuale e sociale si conserva a patto che ci siano condizioni istituzionalizzate per l'apprendimento - uso - verifica delle due lingue e la generazione-espansione delle due culture compresenti. La realtà geo-etnologica del territorio mistilingue dell'Istria, il suo contesto storico e sociale, quello culturale e politico offrono gli elementi di base affinché si stabilisca una sovrastruttura sociale bilingue e biculturale. Questi elementi possono essere articolati in tre gruppi.

1. Nella realtà geoculturale coesistono due lingue/culture autoctone. Bilinguismo e biculturalismo sono le due componenti del campo linguistico e culturale. Esse rappresentano la tradizione, il modo di vivere e la continuità sociale.

2. La politica dell'educazione, definita da leggi specifiche, riflette i bisogni sociali e convalida la realtà.

3. Il contesto sociale si ritrova e riformula grazie allimpegno dei singoli. La volontà sociale riflette l'attività individuale. $\mathrm{La}$ società bilingue si riconosce nel funzionamento di istituzioni governative, economiche e culturali nonchè nell'attività individuale. La scuola è un fattore formativo della società bilingue e transculturale.

Un'analisi valutativa e oggettiva della struttura sociale dell'Istria detta "bilingue" ci dimostra che le condizioni di base, pur essendo favoreveli, non portano agli obiettivi sociali, culturali e politici preposti.

1. Le lingue/culture non si trasmettono bidirezionalmente. La lingua/cultura maggioritaria si propaga verso il gruppo minoritario, la trasmissione inversa è debole. L'unidirezionalità è condizionata da ambiti linguistici e culturali limitati per la lingua/cultura minoritaria. La socializzazione della lingua/cultura italiana è ristretta, riservata alla scuola e ad alcune attività culturali.

2. La tutela legittimata non garantisce il contenuto ma si limita alle forme organizzative di attività culturale e sociale. I contenuti scolastici per esempio non tras- 
mettono né bisogni della comunità bilingue né adempiono il ruolo formativo specifico.

3. La volontà politica non si estende alla volontà sociale. Fanno eccezione le istituzioni scolastiche e culturali. Ma la scuola maggioritaria è ostacolata da schemi tradizionali nell'organizzazione ed è imprigionata in strutture rigide nella formazione del singolo. L'apertura verso l'interdisciplinarità, verso la ricerca sociale e ambientale, lo studio di due lingue come strumento comunicativo e contenuto conoscitivo non si verificano spesso nella prassi scolastica. I programmi scolastici non corrispondono che in parte ai bisogni dell'educazione al bilinguismo, gli insegnanti non sono sempre sensibili ai valori del bilinguismo e del biculturalismo.

Ne deduciamo che i valori del biculturalismo e/o i pregi del bilinguismo si esaltano a livello dichiarativo e non si ritrovano nella vita sociale, non si trasmettono nell'identità personale. Le condizioni economiche e sociali non permettono una riforma globale dell'educazione bilingue. Le condizioni demografiche non stimolano la formazione di un'identità bilingue e biculturale degli abitanti autoctoni dell'Istria.

\section{LA DIDATTICA DELLA LINGUA ITALIANA COME L2 - SPECIFICITÀ}

\section{Dal gioco alla parola}

L'acquisizione della lingua seconda è di solito per i bambini del gruppo nazionale maggioritario successiva all'acquisizione della lingua materna. Per i bambini del gruppo nazionale minoritario l'acquisizione delle due lingue è il più delle volte simultanea. L'ambiente maggioritario gli fornisce dalla prima infanzia in poi elementi e stimoli per l'acquisizione della seconda lingua. Affinchè i bambini del gruppo nazionale maggioritario diventino anche loro bilingui, dal $1980 \mathrm{i}$ programmi dell'educazione prescolare istituzionalizzata del Litorale mistilingue includono l'insegnamento della lingua italiana. Non è un insegnamento vero e proprio. Nel momento in cui il bambino si inserisce nel gruppo di coetanei dell'asilo, esso incomincia ad allargare le conoscenze sul mondo sociale, culturale, linguistico con rapporti, contenuti e codici di tipo non presente nella sua famiglia, ma compresente nel mondo che lo circonda. Conoscere dunque l'italiano nell'asilo è allargare le conoscenze sulla realtà sociale. La cosa è certamente diversa per $\mathrm{i}$ bambini che crescono nelle famiglie plurilingui e plurinazionali. Come i bambini del gruppo nazionale minoritario anche questi bambini sono di solito bilingui dalla tenera età.

Non è pero nell'intento dell'autrice definire le molteplici situazioni linguistiche in cui, per via spontanea o guidata, il bambino del territorio mistilingue dell'Istria diventa parlante bilingue. La sua intenzione è di osservare come il bambino sloveno risponde all'introduzione della lingua italiana nell'educazione prescolare, di trattare l'approccio didattico di questa educazione. 
Dopo aver considerato teorie evolutive e psicodinamiche che sostengono l'acquisizione precoce di una seconda lingua, osservato le condizioni psicofisiche del bambino che favoriscono una tale educazione, dopo aver studiato vari progetti di educazione bilingue altrove e preso in considerazione $i$ risulati delle loro analisi si può tratteggiare una strategia didattica che corrisponda alla situazione educativa specifica. La pianificazione, l'organizzazione e la presentazione della lingua seconda al livello prescolare devono seguire concetti basilari della psicologia e della pedagogia prescolare, nonchè partire dalla glottodidattica moderna e quindi incorporare nei procedimenti didattici le condizioni specifiche. L'acquisizione bilingue prescolare deve essere:

-parte integrante della socializzazione del bambino,

-interdisciplinare, cioè globale,

-spontanea in confronto al bambino, pianificata riguardo all'iter didattico.

Essa deve osservare le condizioni pedagogiche di base:

-L'apprendimento si svolge tramite attività ludiche;

-Il contenuto linguistico si articola in atti linguistici e moduli comunicativi corrispondenti ai bisogni linguistici del bambino;

-La valutazione del feedback linguistico è globale.

Il gioco (cioè qualsiasi forma di attività ludica) è presente in tutte le fasi del processo educativo. Esso attira il bambino all'ascolto, lo coinvolge nel procedimento comunicativo, lo stimola a prender la parola, lo ripaga dell'intervento, lo sensibilizza a conformarsi ai collocutori, lo invita a creare moduli comunicativi.

La lingua seconda si presenta come strumento comunicativo- espressivo e come fattore evolutivo della competenza linguistica in generale. I risultati dell'educazione bilingue (coinvolgimento del bambino nella comunicazione, $i$ suoi interventi) si manifestano così nella possibilità di comunicare nella $\mathrm{L}_{2}$, come nel modo in cui il bambino osserva la seconda cultura, e nelle manifestazioni del suo atteggiamento personale verso il mondo circostante. La valutazione degli effetti educativi non si limita all'analisi degli interventi verbali.

La ricerca empirica ha convalidato l'acquisizione precoce della seconda lingua, c'è però dello scetticismo sulla sua applicazione istituzionalizzata. Le si rimprovera una labile strategia didattica e una scarsa effettività poiché essa puo essere per il bambino un sovraccarico sproporzionato. Il secondo dei due rimproveri è infondato poiché si è gia parlato della possibilità del bambino di addattarsi allo sforzo. E più fondata la costatazione che le strategie didattiche per l'educazione bilingue precoce siano poche e male elaborate.

Negli asili del Litorale si è introdotto l'italiano come L2 nel 1980. La strategia didattica si basa su concetti pedopsicologici e pedagogici già nominati ed e più $\mathrm{o}$ meno originale e specifica. Affinché si potessero provare gli effetti positivi di tale 
strategia o approccio si è creato uno schema d'osservazione con cui si è potuto seguire un gruppo di 23 bambini per un trimestre.

Dopo aver annotato le loro reazioni verbali e non verbali agli stimoli comunicativi, definito il grado qualitativo dei loro interventi verbali, analizzato la forma e il contenuto linguistici e la loro coerenza alla situazione/contesto comunicativi si è potuto costatare:

- Gli atti linguistici acquisiti nell'ambito delle funzioni linguistiche referenziale ed espressiva sono di notevole frequenza, qualità e coerenza, meno soddisfacenti invece nell'ambito della funzione conativa.

- La frequenza degli interventi verbali, la qualità dei moduli comunicativi, il dinamismo delle interazioni verbali e sociali provano che c' è motivazione a una tale educazione e che l'effetto è positivo.

- La mancata frequenza di atti linguistici più complessi prova che l'educazine linguistica al livello prescolare è troppo guidata, dunque limitata.

- Comparando $\mathrm{i}$ dati qualitativi degli interventi linguistici dei bambini con $\mathrm{i}$ risultati dell'analisi conoscitiva degli osservatori costatiamo che la considerazione degli effetti comunicativi verbali non da una valutazione completa. Bisogna creare strumenti di osservazione per tutti gli interventi espressivi nonché valutare lo sviluppo linguistico globale del bambino sottoposto all'educazione bilingue e compararlo con la valutazione dello sviluppo linguistico dei bambini monolingui.

\section{Uso e consapevolezza linguistici}

L'interesse a riprodurre moduli linguistici della $\mathrm{L}_{2}$ e la capacità innata del bambino di sistemarli similmente alla $\mathrm{L}_{1}$ in una definita cornice mentale, ha favorito l'acquisizione di nozioni linguistiche ed ha attivato la motivazione all'apprendimento successivo della seconda lingua. Nell'asilo la motivazione alla $L_{2}$ esiste e si conserva pure nella scuola elementare se l'alunno può convalidare l'appreso nelle situazioni extrascolastiche. Per molti alunni il processo d'acquisizione della $\mathrm{L}_{2}$ inizia e termina in classe. La mancata prassi comunicativa non appaga il loro impegno e non sostiene l'insegnamento dell'italiano. Il bambino dell'età prescolare ha intuito, prima per la lingua madre e poi per la $\mathrm{L}_{2}$, che la lingua è un sistema regolato. Guidato da procedimenti di classificazione, di trasformazione, di complementazione ed altri farà suo il modo di sistemizzare elementi linguistici e acquisirà consapevolezza linguistica. Se la prassi comunicativa si alterna alla sua intuizione/riflessione sulla lingua, essa interiorizzerà i procedimenti comunicativi e ristimolerà la riflessione e l'uso linguistici.

La specificità del Litorale poggia sulla presenza e sul dinamismo di due lingue/culture. Elementi linguistici e culturali complementari e non complementari alla specificità sociale autoctona, di radice e contenuto diversi, spezzano la continuità $e$ bloccano i processi d'integrazione culturale e sociale.

La lingua madre degli alunni della scuola con lingua d'insegnamento slovena non è sempre lo sloveno. Spesso esso si impone come la loro seconda lingua. La preconos- 
cenza linguistica e comunicativa dell'italiano e di diversi livelli è di diversa qualità. Gli alunni che nei processi di acculturazione non hanno interiorizzato valori e nozioni del biculturalismo e del bilinguismo sono insensibili all'educazione specifica. L'ambiente familiare non sostiene sempre l'educazione scolastica, l'ambiente sociale ristretto dell'alunno non stimola le relazioni comunicative che esso desidera instaurare.

Quanto eterogenea sia la struttura delle classi riguardo la lingua madre degli alunni e quanto differenziata sia la loro motivazione al bilinguismo definiscono due analisi di ricerca effettuate nelle scuole del Capodistriano. I test diagnostici somministrati dal 1981 al 1985 a tremila alunni delle $7^{\mathrm{e}}$ classi della scuola elementare provano che esistono almeno tre gradi di classificazione degli alunni corrispondenti a tre livelli della loro competenza linguistica:

a) Il primo gruppo è composto da alunni provenienti da famiglie autoctone, o trasferitesi dal tempo della nascità dei loro figli nel Litorale. La loro lingua madre è lo sloveno, raramente l'italiano o il serbocroato. Gli alunni sono bene motivati allo studio dell'italiano.

b) Il secondo gruppo consiste in bambini di famiglie di immigrati per lo più Sloveni dell'interno. Spesso hanno delle difficoltà nello studio dell'italiano, però cercano di migliorare perché sono motivati ad inserirsi nel nuovo ambiente.

c) Nel terzo gruppo ci sono i figli degli immigrati recenti. Il sistema scolastico e l'ambiente bilingue gli sembrano inconsueti. Si muovono nel gruppo dei pari e dimostrano indifferenza allo studio dell'italiano. Il loro problema principale è la scarsa competenza della lingua d'apprendimento, cioè dello sloveno.

La preconoscenza dell'italiano, lo sviluppo linguistico della lingua madre, la motivazione e l'attitudine allo studio dell'italiano, gli stimoli sociali all'uso di esso sono dunque $\mathrm{i}$ fattori che determinano i livelli di partenza. Un fattore molto importante del processo d'acquisizione della lingua seconda è la strategia didattica dell'apprendimento di questa lingua. Essa deve considerare i diversi livelli, differenziare i contenuti e i metodi, presentare i procedimenti, programmare gli iter didattici individuali e comuni. La base dell'approccio differenziato è l'indagine sugli errori. Essa si svolge al livello di classe tramite test diagnostici, al livello individuale tramite un'osservazione accurata degli alunni mentre effettuano atti linguistici.

Prima di scegliere la strategia correttiva bisogna analizzare le cause degli errori e trovare i motivi che li hanno provocati. Essi derivano dalla personalità dell'alunno (intelligenza generale e specifica, attitudine allo studio delle lingue, sviluppo linguistico, motivazione allo studio), dal lavoro dell'insegnante, (competenza professionale e didattica, motivazione all'insegnamento, il ruolo che l'insegnante assume: insegnantemodello, insegnante-giudice, insegnante-animatore) o dalla situazione didattica in cui l'insegnamento dell' italiano viene posto (interferenze linguistiche, motivazione sociale, qualifica dell'insegnante, programmi, contenuti).

La tipologia degli errori ci interessa in quanto presenta la selezione di un numero limitato di forme scorrette, ma tipiche e frequenti. Gli errori possono essere: 
di competenza (mancata assimilazione di strutture linguistiche)

\section{di produzione o esecuzione (sviste)}

dovuti all'interferenza linguistica (divergenze $\mathrm{L}_{1} / \mathrm{L}_{2}$ ).

Le sviste si eliminano tramite l'autocorrezione. Nell'indagine sugli errori l'insegnante darà peso a quelli dovuti alla mancata riflessione linguistica o a quelli dovuti all'interferenza e ne studierà le cause, proporrà procedimenti correttivi.

La mancata riflessione linguistica si verifica nell'incapacità di realizzare enunciati, costruire proposizioni, formulare concatenazioni o relazioni sul piano grammaticale. Si tratta dell'assimilazione di strutture sull'asse sintagmatico. Gli esercizi di tipo sintagmatico sono considerati prioritari, essi affrontano il problema di scelte obbligate. Gli errori di lessico dimostrano l'incapacità di selezione, di sostituzione di elementi nella frase. Scegliendo un elemento piuttosto di un altro dimostriamo come abbiamo assimilato le nozioni liguistiche sull'asse paradigmatico. Gli esercizi di tipo paradigmatico arrivano dopo che le strutture grammaticali essenziali siano state sufficientemente assimilate.

L'autrice della tesi propone alcuni esercizi correttivi-tipo dedicati all'assimilazione di strutture su ambedue gli assi del sistema linguistico. Le batterie di esercizi siano ad uso individuale o di piccolo gruppo. La strategia correttiva parte dall'errore e propone il lavoro differenziato riguardo alle cause che lo hanno provocato. I passi che determinano il ritmo e la velocità del procedimento correttivo si definiscono individualmente. Il feedback è autocontrollato, esso permette o blocca la continuazione dell'esercitazione. Il passaggio alla successiva sequenza della batteria dipende dalla riuscita della sequenza precedente.

\section{La seconda lingua - strumento d'interazione linguistica e sociale nell'ambiente bilingue}

L'acquisizione della seconda lingua si effettua tramite il processo didattico e tramite interazioni personali e sociali dell'alunno sloveno con singoli e comunità parlanti l'italiano. La radio, la televisione, la stampa, le comunicazioni informali arricchiscono l'imput in italiano dell'alunno. Se in asilo i primi approcci alla seconda lingua destano la curiosità del bambino e lo invogliano a riprodurre moduli, se la scuola elementare tramite nozioni apprese allarga la possibilità dell'alunno di comunicare e gli fa riflettere sulla norma linguistica dell'italiano la scuola media gli deve offrire le condizioni in cui egli verifichi l'effetto dell'atto comunicativo nella seconda lingua. Non bastano più le situazioni simulate e fittizie dell'insegnamento linguistico in classe. Il procedimento didattico deve svolgersi nella ricerca di ambiti liguistici autentici in cui la conoscenza dell'italiano si dimostra come necessità concreta dell'alunno. L'insegnamento si sposta dalla scuola alla vita, i bisogni linguistici si realizzano in situazioni extrascolastiche. 
L'apprendimento linguistico a scuola è un processo guidato, prende spunto dall'intelligenza, dalla motivazione e si consolida tramite la riflessione e l'uso linguistici, si interiorizza diventando con l'aiuto dell'esperienza competenza linguistica e comunicativa. I fattori che influenzano l'apprendimento linguistico sono determinati dall'ambiente scolastico e sociale. La scuola contribuisca dunque a creare l'opportunità in cui vivere tramite l'espressività, il simbolismo, la concretizzazione linguistici l'esperienza del singolo in rapporto con la società.

Tra $\mathrm{i}$ metodi e le tecniche didattiche che rispondono di più all'esigenza di favorire lo sviluppo mentale tramite attività euristiche, rafforzano l'intuizione e la riflessione, evolvono la capacità di risolvere problemi e assimilare gli strumenti per l'esperienza è il metodo di ricerca o il procedimento ricerca- azione.

Far ricerca nell'ambito dell'insegnamento linguistico non significa fare qualcosa di diverso per non fare la solita lezione di grammatica. Essa è una tecnica di insegnamento linguistico funzionale, riguarda la sfera pratica dell'uso della lingua, rende operativa la norma linguistica e si articola in una particolare struttura mentale e logica in cui si imposta un problema. Attraverso il lavoro individuale l'alunno riesce a organizzare le sue conoscenze cognitive, accresce la sua cultura, si fa schemi operativi applicabili a nuove indagini conoscitive, culturali e linguistiche.

Il lavoro di ricerca coinvolge gli alunni, gli insegnanti ed altri operatori didattici. Nella ricerca-metodo d'insegnamento l'insegnante svolge la sua attività rinunciando alla supremazia dell'esperto. Egli si mette sullo stesso piano dell'alunno, contribuendo da esperto con strumenti e procedure alla soluzione del problema. Segue, raddrizza e corregge il procedimento didattico laddove gli alunni non riescono a procedere da soli.

Gli alunni arrivano al traguardo con risultati diversi. Ciascun alunno compie il proprio iter d'acquisizione in base alle proprie possibilità. La valutazione degli effetti linguistici, cognitivi e educativi è intesa come momento formativo, come controllo dell'acquisito e come regolazione degli obiettivi dell'apprendimento futuro.

Nell'anno scolastico 1984/85 tre scuole medie superiori del Capodistriano (6 classi e 3 insegnanti) hanno riformato il metodo d'insegnamento della lingua italiana alternando il lavoro tradizionale con la ricerca ed altre tecniche attive ed euristiche (tavole rotonde, recital in classe, corsi compatti monografici).Le scuole pilota hanno reorganizzato l'insegnamento dell'italiano con procedimenti metodologici interdisciplinari ed extrascolastici. Si proponevano dunque delle strategie didattiche meno tradizionali con cui presentare i contenuti dell'insegnamento linguistico. Affinché l'aspetto didattico applicativo potesse realizzarsi, c'era necessità di:

-definire il repertorio di contenuti specifici,

-prestare l'insegnamento a forme nuove d'insegnamento (interdisciplinari, extrascolastiche, intensive),

-aggiungere al lavoro di ricerca, alle tavole rotonde, ai recital elementi educativi, provenienti dal lavoro stesso. 
In parte queste strategie didattiche presentano metodologie innovative. Basandosi sulla tradizione, arricchita di esperienze vissute dagli allievi e dai loro insegnanti, sulla flessibilità dell'organizzazione e dei procedimenti, si superano $i$ formalismi tradizionali della vecchia scuola.

Attraverso l'osservazione e l'analisi dell'ambiente, le ricerche bibliografiche, le testimonianze e i documenti gli alunni approfondiscono la conoscenza dei valori culturali e sociali. Alla luce di nuove strategie didattiche la narrativa, le attività letterarie e linguistiche ed altri valori culturali diventano materia d'indagine e allo stesso tempo materia conoscitiva. Qualche esempio illustrerà meglio il procedimento.

Le opere letterarie di Fulvio Tomizza sono la sintesi delle sue radici istriane e della matrice culturale italiana. Per le sue tonalità squisitamente istriane, inserite nella vasta cultura italiana sono l'ideale per esser presentate a chi questa duplice cultura la subisce e la vive. Vengono dunque presentati in classe singoli capitoli, spezzoni di romanzi del nostro autore che servono all'elaborazione linguistica e socioculturale. Dopo aver apprezzato esteticamente e linguisticamente la narrativa di Tomizza si procede all'indagine: confrontare il messaggio letterario, esteriore, altrui, con la realtà vissuta di una cultura autoctona; mettere in moto il processo di ricerca, di apprezzamento, di valorizzazione; coinvolgere i giovani rendendoli partecipi nel sentire questa comune cultura.

Il discorso sui letterati di lingua italiana dell'Istria interessa in primo luogo gli uomini di lettere, ma deve coinvolgere gli strati e le istituzioni vitali in cui essi operano. Introducendo nell'insegnamento della lingua italiana come $\mathbf{L}_{2}$ forme atte a socializzare la letteratura del gruppo nazionale dell'Istria significa incaricare i giovani di gestire il patrimonio culturale che spetta loro. Ricerche bibliografiche, tavole rotonde, interviste ed incontri con poeti, narratori, musicisti contribuiscono all'apprendimento di una lingua, vista nel suo contesto culturale.

L'ambiente bilingue si dimostra tale se e quando porta l'impronta di regolamentazione bilingue (scritte topografiche e informative, comunicazione ufficiale e pubblica, ecc.) Nelle ricerche sul bilinguismo visivo gli alunni possono controllare l'efficienza, la qualità e la quantità dei regolamenti che lo stabiliscono. Le loro costatazioni e le loro proposte diventano valide quando destano l'interesse di chi è responsabile di regolare la comunicazione bilingue nell'ambiente sociale.

L'innovazione introdotta negli anni 1984/85 viene così articolata:

determinazione degli obiettivi

scelta degli argomenti

tappe di lavoro (organizzazione, forme di lavoro, mezzi didattici)

proposte operative

valutazione dell'innovazione secondo la metodologia proposta.

Si è cercato di stabilire e analizzare gli effetti del periodo di sperimentazione con l'aiuto di due questionari (per gli alunni e per gli insegnanti), di relazioni sulla ricerca 
preparate dagli alunni-ricercatori, di interviste avute dall'autrice con gli insegnanti fuori sperimentazione e con i direttori delle scuole. L'elaborazione di dati, risposte, documenti ci ha portati ad alcune costatazioni.

La motivazione per lo studio dell'italiano è accresciuta, è aumentato l'uso attivo della $L_{2}$ degli alunni, essi hanno appagato $i$ loro interessi avendo scelto o aiutato a scegliere i contenuti e le tecniche di lavoro. Il loro lavoro autonomo è stato producente e qualitativo. Gli insegnanti si preoccupavano di non aver potuto controllare l'acquisizione del sistema linguistico e di non aver seguito a sufficienza la competenza della comunicazione scritta in lingua italiana. La situazione didattica cambiata ha portato vantaggi soprattutto agli alunni, gli insegnanti si sono presi maggior responsabilità siccome il processo di acquisizione dipendeva più che mai dal ruolo attivo degli alunni. L'interazione di relazioni, di contenuti e di effetti ha scompigliato gli schemi mentali di chi era legato al tradizionale e alla routine scolastica.

I contenuti linguistici, culturali e sociali dell'ambiente bilingue si sono offerti all'indagine degli alunni diventando materia autentica di riscontro dei loro interessi.

\section{BIBLIOGRAFIA}

Boljun-Debeljuh L.: 1985, Proučavanje stavova prema dvojezičnosti i prema jezicima i govorcima jezika društvene sredine uz određivanje tipologije upotrebe jezika, Filozofska fakulteta Zagreb, neobjavljeno magistrsko delo

Borgia Charlotte: 1983, Vers une éducation interlinguistique et transculturelle, Atti del convegno internazionale, Mantova

Bugarski. R.-Mikeš M.: 1984, Pregled tipova i metoda istraživanja višejezičnosti u Jugoslaviji, Dvojezičnost individualne in družbene razsežnosti, Ljubljana (117-127)

Combi F.: 1977, La ricerca in pedagogia, Piccola enciclopedia di scienze dell'educazione, Le Monnier,Firenze

Cummins J.: 1984 a, Bilingualism and Special Education: Issues in Assessments and Pedagogy, Multilingual Matters, Cleveland, England

Čok L.: 1983, Verso un'educazione biculturale e bilinguistica, Voce del popolo, $8 / 9$, Edit Rijeka 
Čok L.: 1986, La ricerca didattica nell'insegnamento della lingua italiana (I valori della nazionalità italiana come contenuti didattici), Scuola nostra 17 - 18, Edit Rijeka

Č́ok L.: 1986, L'éducation bilingue et transculturelle dans les écoles du Littoral Slovène - particularité de l'orientation didactique, Razprave in gradivo 18, Inštitut za narodnostna vprašanja, Ljubljana

Fishman J. A.: 1978, Sociologija jezika, Igkro "Svjetlost" Sarajevo

Francescato G.: 1973, Il linguaggio infantile. Strutturazione e apprendimento, Torino, Enaudi

Francescato G.: 1981, Il bilingue isolato, Minerva italica, Roma

Grassi C.: 1986, Il bilinguismo infantile e le sue implicazioni sociolinguistiche e scolastiche, Parma, Atti del convegno IRRSAE Emilia-Romagna, L'Educazione Logico Linguistica nella scuola Primaria

Girard D.: 1977, Le lingue vive e il loro insegnamento, Editrice La Scuola, Brescia

Katerinov K.: 1975, L'analisi contrastiva e l'analisi degli errori di lingua applicata all'insegnamento dell'italiano, Rassegna di Linguistica applicata, Anno VII, n 2-3

Klinar P.: 1980, Procesi socializacije in etnične identifikacije, Teorija in praksa 5, str. 540

Kunst-Gnamuš O.: 1983, Govorno dejanje - družbeno dejanje, PI pri Univerzi E. Kardelja, Ljubljana

Lado Robert: 1964, Language Teaching, A Scientific Approach, McGraw - Hill Inc. New York

Mackay W. F.: 1982, L'apprendimento precoce scolastico ed extrascolastico della seconda lingua: rapporti tra bilinguismo e didattica delle lingue, Atti del convegno internazionale, Bolzano

McLaughlin B.: 1978, Second Language Acquisitation in Children, Hillsdale, Erlbaum 
Milani-Kruljac N.: 1985, La comunità italiana in Jugoslavia tra diglossia e bilinguismo, Filozofska fakulteta Zagreb, neobjavljena doktorska dizertacija

Pečjak Vid: 1975, Psihologija spoznavanja, DZS Ljubljana

Prunč E.: 1986, Problemi zgodnje otroške dvojezičnosti, Slovenski tednik št. $7-25$, Celovec

Sečrov Neva: 1984, Risultati di un sondaggio, Scuola Nostra 14, Edit Rijeka

Slama-Cazacu T.: 1982, Rilevanza della concezione sul bilinguismo precoce per l'identificazione dei fattori che ne facilitano lo sviluppo ottimale, Bolzano, Atti del convegno internazionale, 139-161

Strmčnik F.: 1978, Sodobna šola v luči programiranega pouka, DDU Univerzum, Ljubljana

Titone R.: 1971, Bilinguismo precoce e educazione bilingue, Armando Editore, Roma

Titone R.: 1986, "Ragioni di una educazione bilingue precoce e prospettive didattiche". Lingue e culture minoriterie nella scuola italiana, CEDE, Roma

Weinreich U.: 1974, Lingue in contatto, Boringhieri, Torino

Wilkins A.: 1973, Linguistica e insegnamento delle lingue, Zanichelli

Povzetek

\section{PRISPEVEK K DIDAKTIKI ITALIJANSKEGA JEZIKA KOT DRUGEGA JEZIKA}

V magistrski nalogi avtorica predstavlja teoretična izhodišča izobraževanja za dvojezičnost v svetu in pri nas, na obalnem območju Slovenije. Italijanski jezik kot drugi jezik v obalnih šolah ni le učni predmet; predstavlja enega od jezikov družbenega okolja in eno od prvin mešane slovensko-romanske avtohtone kulture. Poučevanje italijanskega jezika se zato mora razlikovati od pouka tujih jezikov. V nalogi so razčlenjene temeljne didaktične značilnosti, ki s predlogi uresničitve poskušajo oblikovati samobitno orientacijo pouka italijanskega jezika kot drugega jezika. Naloga ima v originalu tri poglavja: Teoretične osnove dvojezičnosti, dvojezičnosı v življenjskem okolju in v šoli, Prispevek k didaktiki italijänskega jezikal - jezika družbenega okolja in Izobraževanje za dvojezičnost v pedagoški praksi. Dopolnjujejo jo priloge o empirični zasnovi predlogov in delovni materiali. Prispevek $v$ tej reviji je skrajšana vsebina prvih dveh poglavij. 